\title{
The autonomous vision system on TeamSat
}

\author{
Betto, Maurizio; Jørgensen, John Leif; Riis, Troels
}

\section{Published in:}

Proceedings of IEEE Aerospace Conference

Link to article, DOI:

10.1109/AERO.1999.793148

Publication date:

1999

Document Version

Publisher's PDF, also known as Version of record

Link back to DTU Orbit

Citation (APA):

Betto, M., Jørgensen, J. L., \& Riis, T. (1999). The autonomous vision system on TeamSat. In Proceedings of IEEE Aerospace Conference (Vol. 2, pp. 83-93). IEEE. https://doi.org/10.1109/AERO.1999.793148

\section{General rights}

Copyright and moral rights for the publications made accessible in the public portal are retained by the authors and/or other copyright owners and it is a condition of accessing publications that users recognise and abide by the legal requirements associated with these rights.

- Users may download and print one copy of any publication from the public portal for the purpose of private study or research.

- You may not further distribute the material or use it for any profit-making activity or commercial gain

- You may freely distribute the URL identifying the publication in the public portal

If you believe that this document breaches copyright please contact us providing details, and we will remove access to the work immediately and investigate your claim 


\section{The Autonomous Vision System On TeamSat}

\author{
Maurizio Betto \\ Technical University of Denmark \\ Department of Automation \\ Bld. 327 \\ 2800 Lyngby \\ Denmark \\ +45253439 \\ mbe@iau.dtu.dk
}

\author{
John L. Jørgensen \\ Technical University of Denmark \\ Department of Automation \\ Bld. 327 \\ 2800 Lyngby \\ Denmark \\ +45253448 \\ ili@iau.dtu.dk
}

\author{
Troels Riis \\ Technical University of Denmark \\ Department of Automation \\ Bld. 327 \\ 2800 Lyngby \\ Denmark \\ +45253439 \\ tr@iau.dtu.dk
}

\begin{abstract}
The second qualification flight of Ariane 5 blasted off the European Space Port in French Guiana on October 30, 1997, carrying on board a small technology demonstration satellite called TeamSat. Several experiments were proposed by various universities and research institutions in Europe and five of them were finally selected and integrated into TeamSat, namely FIPEX, VTS, YES, ODD and the Autonomous Vision System, AVS, a fully autonomous star tracker and vision system. This paper gives a short overview of the TeamSat satellite; design, implementation and mission objectives. AVS is described in more details. The main science objectives of the AVS were to verify, in space, multiple autonomous processes intended for spacecraft applications such as autonomous star identification and attitude determination, identification and tracking of non-stellar objects, imaging and real-time compression of image and science data for further ground analysis. AVS successfully determined the attitude and attitude dynamics of TeamSat.
\end{abstract}

\section{TABLE OF CONTENTS}

\author{
1. BACKGROUND \\ 2. ORGANIZATION AND SCHEDULE \\ 3. TEAMSAT OVERVIEW \\ 4. THE EXPERIMENTS ON-BOARD TEAMSAT \\ 5. THE AUTONOMOUS VISION SYSTEM \\ 6. THE AVS MISSION \\ 7. MISSION RESULTS \\ 8. CONCLUSIONS \\ 9. AKNOWLEDGEMENTS \\ 10. REFERENCES \\ 11. BIOGRAPHY
}

\section{BACKGROUND}

The second qualification launch of the Ariane 5 had to be a full scale flight injecting into GTO with two dummy bodies representing typical Ariane 5 payloads. The two dummies, dubbed MAQSAT-H and MAQSAT-B (Maquette Satellite = dummy satellite in french), were two large and heavy, but pretty empty, structures carring sensors to measure the vibrations, shock and acoustic noise during the ascent. The measurements, recorded during this phase, had to be transmitted to ground soon after the separation from the Ariane 5. Therefore, there was space to add other

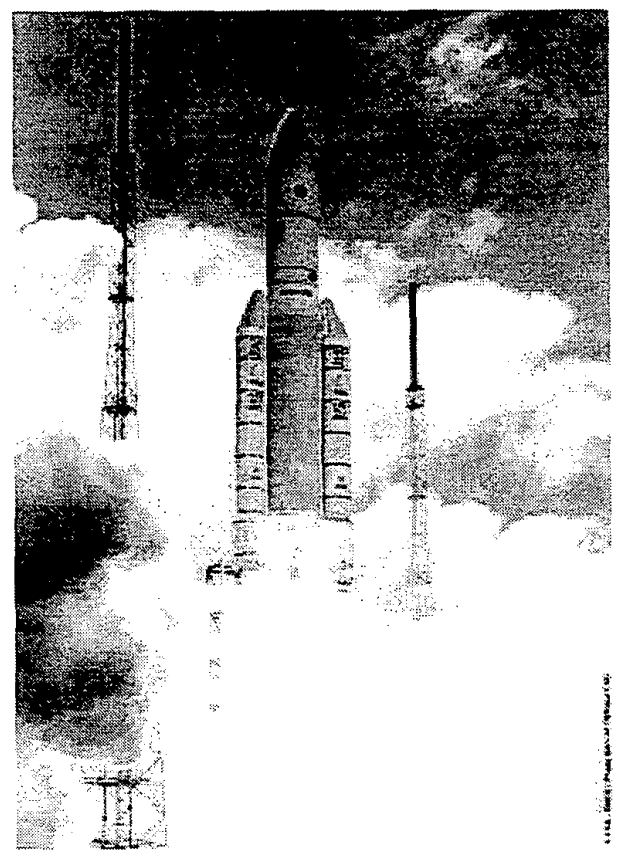

Figure 1: Ariane 502 blasting-off from the launch pad in Kourou (French Guyana)

experiments and this concept, concived by the Ariane 5 APEX Office, was endorsed by the ESA Council held on the $26^{\text {th }}$ of October 1996 but the experiments had to be scientific and educational.

The selection of the experiment had to be done very quickly and it had to focuse on experiments that had hardware ready to fly because the planned launch date was the $15^{\text {th }}$ of April 1997. Of the various experiments proposed from different institutes, five of them were selected by the beginning of November. These experiments had to be integrated on the same platform providing the primary resources, namely: power, data handling and telecommunications. The platform and the experiments were called TeamSat for Technology scientific and Educational experiments Added to MAQSAT$\mathrm{H}$ but mainly to express the attitude and commitment expected from the people to tackle such a challenging project. 
To minimize the impact on the Ariane 5 ongoing activities and the modification to the design of MAQSAT-H, it was decided to allocate to TeamSat a mass of $350 \mathrm{Kg}$ and to attach it to the bottom of MAQSAT-H inside the frustum of cone holding MAQSAT-H on the separation ring from the speltra (fig.2).

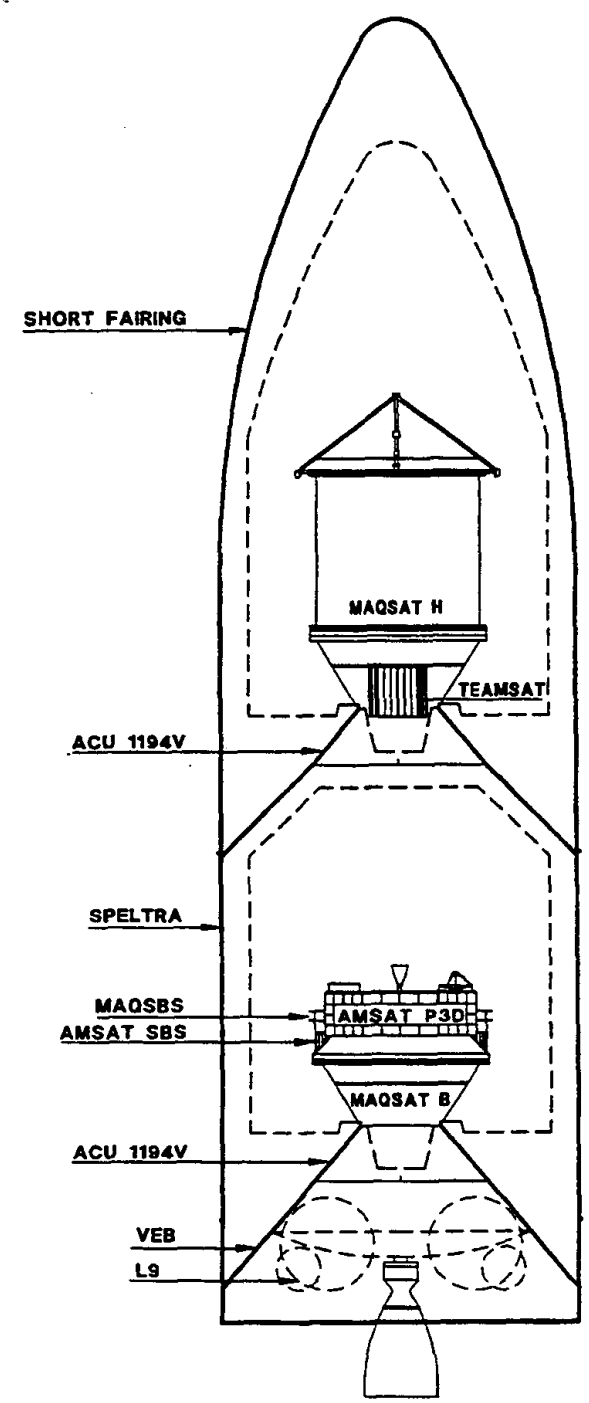

Figure 2: The Ariane 502 launch configuration and the position of TeamSat

\section{ORGANIZATION AND SCHEDULE}

Though the ESA council endorsed the project, the only goal of the A502 launch remained and was the qualification of the launcher. Therefore TeamSat had to meet all the A502 milestones. As the launch was originally planned for the $15^{\text {th }}$ of April 1997, we had only ten working weeks for the design, the development, the integration and the testing of the system. The shortage of time and the very limited funds allocated to TeamSat ( $600 \mathrm{KECU} \approx 720 \mathrm{KUS} \$$ ) did not allow a normal procurement of the components neither a normal management of the project. The KISS (Keep It Simple, Stupid) philosophy was the only suitable approach.

To reduce the costs and to increase the productivity it was decided to reuse as much as possible flight hardware available in the ESTEC labs and storage and to build in house what was possible. Standard interfaces were agreed with the experiments and the design of the On-Board Data Handling (OBDH) started immediately in parallel with the mechanical design of the TeamSat structure. Meanwhile the manufacturing of the experiments' flight hardware began. Three Nickel-Cadmium (NiCd) batteries manufactured in 1983 and preserved at $-8{ }^{\circ} \mathrm{C}$ and two transponders from Olympus and Eureka where found in ESTEC and used. The checkout terminal equipment of Cluster and the in-orbit mission control system based on the ESOC SCOS-II were used and some support was given by external companies eager to validate their products in a real mission. In accordance with the decision of the council, the team gathered senior engineers on the side of students and young graduate engineers for the design, manufacturing and testing of the hardware.

The schedule was pivoting about the mechanical acceptance of the flight hardware for which very stringent requirements were set. Indeed the main concern from the main bodies (ESA, CNES and ARIANESPACE) was not to have the qualification flight jeopardized by TeamSat. As the launch was postponed and the mechanical (vibration, acoustic and shock) tests were successfully passed, the activities started to focus on the electrical and interface testing. The abnormally long test phase allowed detecting and fixing all the small problems that were generated by the very short design and manufacturing phase and by the end of June 1997 TeamSat was ready to leave ESTEC and the $15^{\text {th }}$ of July the launch campaign officially started.

\section{TEAMSAT OVERVIEW}

The very short time and the limited funds available to complete TeamSat forced us to have a very simple satellite. The TeamSat structure was a hexagonal box done in Aluminum divided internally in two parts to host the Team experiments and YES (the experiments are described later in this paper). The mass was $350 \mathrm{Kg}$ and the dimensions were: height $=0.75 \mathrm{~m}$, radius of the circumscribed circle $0.91 \mathrm{~m}$ so to fit inside the cone supporting MAQSAT-H on the separation ring. MAQSAT-H was mainly a $2000 \mathrm{Kg}$ cylinder $4.8 \mathrm{~m}$ long (incl. the cone and the structure on the top) and with a diameter of $2.5 \mathrm{~m}$ (fig. 2).

Inside the box there were the system resources and the experiments' electronic boxes (fig. 3). Whereas the optical heads and the sensors were mounted externally on the cone (fig. 4). 


\section{TEAMSAT Subsystem Classification}

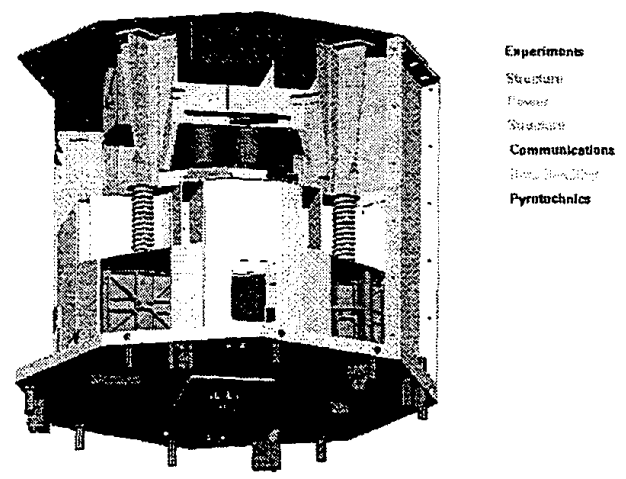

Figure 3: TeamSat subsystems and internal architecture

TeamSat was a slow spinner (0.5-rpm) with no active attitude control system. Some concerns were raised by some experiments, as, despite the geometry, MAQSAT-H spinning axis was the axis of minimum inertia. This meant that TeamSat was intrinsically unstable and that eventually it would have gone in flat spin. However, as there was not energy dissipation on board except for the flexible mode of the structure and the mission could only last few days, some coarse analysis showed that this was not an issue for TeamSat.

The thermal control was also passive and based on conduction and isotropic irradiation inside the box whereas the units mounted outside were covered by MLI.

The mission duration and satellite lifetime were limited by the three NiCd batteries as no solar cells could be mounted on MAQSAT-H and the TeamSat box was inside the cone.

The OBDH was designed and manufactured in ESTEC. It was the first system fully compatible with the ESA/CCDSDS (Consultative Committee for Space Data Systems) standards and the first spacecraft to exploit the adaptive asynchronous TM capabilities they support. The boards and the design were based on the use of ASICs (Application Specific Integrated Circuits). The useful bandwidth available on the TM link was $28.259 \mathrm{Kbits} / \mathrm{sec}$.

The ground segment included two ground stations (Perth Australia and Kourou - French Guyana), the Mission Control System at ESOC in Germany and the Experiment Control Center at ESTEC in The Netherlands. A new approach, which was successfully adopted for TeamSat to reduce the costs, was to integrate the EGSE of the equipment, normally meant only test the units before the launch, into the Mission Control System to monitor the experiments and to gather the data.

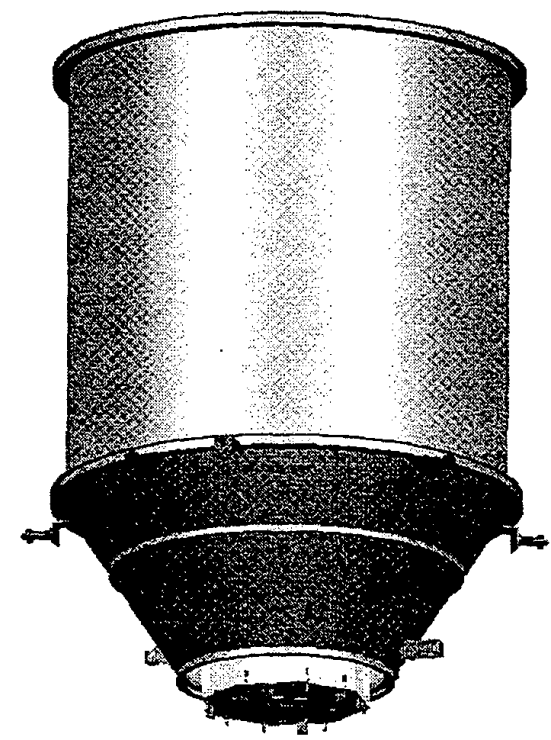

Figure 4: MAQSAT-H and the position of the TeamSat external sensors and optical heads

\section{THE EXPERIMENTS ON-BOARD TEAMSAT}

Five experiments were selected and integrated into TeamSat.

\section{Autonomous Vision System (AVS)}

Designed and developed by the Technical University of Denmark, this is a versatile autonomous star tracker that can recognize and track non-stellar objects like asteroids and other spacecraft's.

\section{Flux Probe Experiment (FIPEX)}

Proposed by the University of Stuttgart (D), it measured the concentration of atomic oxygen up to $1000 \mathrm{Km}$. The perigee passage of the GTO provided a particularly good opportunity to measure altitude profiles of the atomic oxygen concentrations. This experiment included five sensors mounted externally and one electronic box. It was not particularly sensitive to the TeamSat attitude and it was operative only about the perigee.

The interest in having good data about the atomic oxygen distribution near Earth is large in the space community, as it is very corrosive.

FIPEX gathered very valuable data and the mission was a big success for them. The results are being published in specialized journals.

\section{Visual Telemetry System (VTS)}

Initially developed by Matra Marconi Space (UK) to monitor ENVISAT, VTS was proposed by ESTEC and it 
flew for the first time on TeamSat. VTS consists of an electronic unit and several cameras using Active Pixel Sensor (APS). The main advantage of this technology is the very large dynamic range.

The role of VTS on TeamSat was to take images of the opening of the fairing, the separation from the speltra and the ejection of YES.

On TeamSat VTS featured one electronic box and three cameras. Commands to take one or more images could be up-linked. The images were stored on-board in the electronic box to be transmitted upon request. VTS failed to take images of the opening of the fairing probably because the automatic sequence to start the VTS operations was not properly calibrated. However, VTS got an outstanding sequence of images of the separation from the speltra and the mission was considered a great success.

\section{Orbiting Debris Device (ODD)}

Proposed by ESTEC, but involving several other institution and astronomical observatories, ODD was the only completely passive experiment on-board TeamSat. The MAQSAT-H cylindrical body was painted with a high contrast pattern ( $75 \%$ white and $25 \%$ black) using paint of known optical properties to support test and calibration of the ground-based optical and radar stations to be successively used to track the space debris. Being completely passive this experiment could last well beyond the other experiments and it is indeed still running.

\section{Young Engineers Satellite (YES)}

YES was proposed by the Technical University of Delft. This was a micro-satellites tethered system. Mounted on the bottom of TeamSat, YES should have first deployed a 35 $\mathrm{Km}$ tether and then it should have been ejected from TeamSat to re-enter and burn into the atmosphere. YES was to study the tether dynamics and it also included some other experiments on its platform to measure the radiation, the acceleration and the solar angle autonomously after the separation from TeamSat. YES and the deployment of the tethered were controlled by JORIS, a computer completely designed and assembled by young engineers and students. Furthermore, it carried a GPS receiver to evaluate the use and the performance of the GPS at altitudes above the GPS constellation.

Unfortunately, when YES was already assembled, the International Steering Committee for Space Debris gave its no-go decision for the deployment of the tether because they consider too risky the mission. Indeed the launch window did not guarantee the right conditions to have the solar radiation pressure pushing the tether into the atmosphere.

Therefore, it was decided to run a dry mission, i.e. to eject YES (without any tether) and to rehearsal the tether operations. YES was a big success despite the de-scoped mission and the inadvertent ejection of YES which, unfortunately, could be monitored neither by VTS nor by AVS. The system worked properly and the GPS experiment received signals from the GPS satellites at altitude above the GPS constellation.

\section{THE AUTONOMOUS VISION SYSTEM}

\section{Principle of Operations}

The Autonomous Vision System is a versatile and highly autonomous instrument that automatically recognizes the star field and tracks the non-stellar objects (like satellites, comets and asteroids). The AVS can be used as attitude sensor as well as navigation sensor and science camera.

The instrument has been optimized towards supplying the highest possible overall attitude accuracy for the spacecraft. This has been achieved by splitting the instrument into a Camera Head Unit (CHU) and a Data Processing Unit (DPU), which may be separated by more than $20 \mathrm{~m}$. Because each CHU dissipates $0.35 \mathrm{~W}$ only, and as its mass is less than $250 \mathrm{~g}$, the CHU might be placed close to the instrument setting the highest attitude requirements. Furthermore, the thermal dissipation and the thermal radiation to space through the lens is arranged to ensure full performance without active thermal control (Peltier coolers/heaters) for most mission profiles. The DPU might drive a single $\mathrm{CHU}$ at a user selectable update rate from $0.0625 \mathrm{~Hz}$ to $4 \mathrm{~Hz}$; two CHU's from 0.0625 to $2 \mathrm{~Hz}$ or four CHU's from 0.0625 to $1 \mathrm{~Hz}$.

After the CPU gains access to the digitized image, it analyses the star positions in order to calculate the attitude of the boresight and the rotation about this axis. These coordinates are then transformed to a user defined spacecraft coordinate system, and are output in the form of quaternion. The image analysis functions performed on an image depend on several conditions. In normal operation, the angular difference between two consecutive images is so small that the following procedure is adequate. The image is sifted for stars. All detected stars are warped and their positions are calculated with hyperacuity. Based on the previous attitude and the star catalogue, a star image is formed. The two images are then matched. Based on this match, the relativistic attitude is found. This attitude is then corrected for astronomical aberration and output to the telemetry queue. In a number of situations, for example after power cycling, after a SEU, or following invalid images (bright objects etc.), the previous attitude is invalid or missing. In this case an extra image-processing step, initial attitude acquisition, is included. The hyperacuity star positions are analyzed for triplets of nearest and next nearest neighbors. The resulting set of triplets is then matched to a preflight-compiled version of the star catalogue, the star database. The entries in this star database are all conceivable triplets. Based on this match a crude attitude is obtained. This attitude is then used as a bias instead of the previous invalid attitude, in the consecutive processing. In order to transform the relativistic attitude to heliocentric attitude, the velocity vector of the spacecraft relative to the heliocentric 
system is needed. This vector is obtained via the day of year and an orbit model. The orbit model needs to be updated at intervals from hours to days depending on the orbitperturbing forces (air-drag). Typically, these updates are based on GPS data. The correction amounts to maximum 26 arcsecond for LEO.

Apart from the attitude determination, the software maintains a list of other functions, which may be divided into the following three categories:

1. Communication functions: An $\mathrm{I} / \mathrm{O}$ queue for $T M$ and TC. A dedicated debug-line for monitoring and closedloop testing. System and housekeeping monitoring.

2. Supervisory functions: A SW-watchdog monitors the task switching and program execution. A hot spot database that monitors the aging and occasional radiation damages of the $\mathrm{CCD}$-chip. A bit-washing function refreshing the Hamming-code protected memory at user-specified intervals.

3. Imaging functions: Automatic or user specified image acquisition, compression and TM. A planetary featuretracking module. Non-stellar-object detection and tracking.

The fact sheet of the AVS is given in table 1.

\section{Software verification}

The functions and operations of the instrument have been thoroughly tested. The robustness of the system has been assessed by real sky tests. In this way, the performances in areas which, based on simulations, are supposed to present difficulties, either due to stellar distributions far from the average, or to excessive numbers of false stellar objects, such as galaxies, planets, the moon or satellites, have been verified. The code has been proved to operate nominally and yielding a recovery from a lost-in-space condition of $100 \%$ at the entire night-sky. By adding artificial noise-objects to the image prior to analysis it was proven that the algorithms might handle up to 20 false objects without noticeable performance degradation.

Accuracy was also established via real-sky tests. In these tests the camera was mounted on an astronomical telescope acting as a precision pointing platform. Generally, only atmospheric effects limited the accuracy assessment, and this is why the 0.7" RMS noise on the pointing accuracy is a conservative estimate. The overall accuracy over several thousands of measurements, and over several hours (e.g. moving the FOV from galactic low to high), is typically 1 " RMS pointing and 4-5" RMS in roll about the boresight. This is including the changes in the atmospheric refraction and thermal shifts. As the roll accuracy is intrinsically less than the accuracy in pointing, to improve the overall accuracy and to increase the robustness towards blinding, the simultaneous operation of two CHU's has been analyzed in real-sky tests. Using two CHU's, an equal accuracy of $0.7 "$ for the three axes was established, and a smooth transition from one to two CHU's and back was proven
Table 1: AVS fact sheet

\begin{tabular}{|c|c|c|}
\hline Size & $\mathrm{CHU}$ & $\begin{array}{l}50 \times 50 \times 54 \text { incl. lens, exclusive } \\
\text { baffle }\end{array}$ \\
\hline & $\overline{\mathrm{DPU}}$ & $\begin{array}{l}100 \times 100 \times 102 \text { incl. mounting } \\
\text { flanges }\end{array}$ \\
\hline \multirow[t]{3}{*}{ Mass } & $\mathrm{CHU}$ & $252 \mathrm{~g}$ incl. 30KRad shielding \\
\hline & DPU & $907 \mathrm{~g}$ incl. 30KRad shielding \\
\hline & Std baffle & $60+270$ g exclusive bolts \\
\hline \multirow[t]{2}{*}{ Temperature } & $\mathrm{CHU}$ & $-40 /+20 \mathrm{C}$ \\
\hline & $\overline{\text { DPU }}$ & $-20 /+70 \mathrm{C}$ \\
\hline \multirow[t]{2}{*}{ Voltage } & $\mathrm{CHU}$ & generated by DPU \\
\hline & $\overline{\mathrm{DPU}}$ & $\begin{array}{lll}\text { unregulated } & \text { voltages } & \text { from } \\
16.8 \text { to } 75 \mathrm{~V} & & \\
\end{array}$ \\
\hline \multirow[t]{2}{*}{ Power } & $\mathrm{CHU}$ & $0.6 \mathrm{~W}$ \\
\hline & DPU & $7 \mathrm{~W}$ \\
\hline Comm. & DPU & $\begin{array}{l}\text { RS } 422 \text { full duplex and user } \\
\text { selectable bits }\end{array}$ \\
\hline Precision & \multicolumn{2}{|c|}{$\begin{array}{l}\text { Single image accuracy is better than } 1 \\
\text { arcsecond } 1 \sigma \text { pointing and } 5 \text { arcsecond } 1 \sigma \\
\text { roll. Further improvements based on an } \\
\text { orbital model and multiple images are } \\
\text { possible. }\end{array}$} \\
\hline Update rate & \multicolumn{2}{|c|}{$\begin{array}{l}1 \text { Full precision update per second. Attitude } \\
\text { delay is less than } 2 \text { seconds. Time aperture } \\
\text { jitter less than } 1 \mathrm{~ms} \text {, with respect to e.g. GPS } \\
\text { time. The update rate may by command be } \\
\text { from } 1 / 16 \text { to } 5.5 \mathrm{~Hz} \text { at slightly reduced } \\
\text { accuracy. }\end{array}$} \\
\hline Autonomy & \multicolumn{2}{|c|}{$\begin{array}{l}\text { Auto system gain setting. Fast autonomous } \\
\text { recovery from optical overloading. } \\
\text { Autonomous recovery from lost in space in } \\
300 \mathrm{mS} \text {. Auto imaging with selectable } \\
\text { compression. Auto non-stellar object } \\
\text { tracking. Autonomous single-multiple CHU } \\
\text { operation switching. }\end{array}$} \\
\hline $\begin{array}{l}\text { Star } \\
\text { catalogue }\end{array}$ & \multicolumn{2}{|c|}{$\begin{array}{l}\text { Stellar positions based on a compilation of } \\
\text { Hipparcos data and PPM intensities. The } \\
\text { AVS catalogue contains the } 13.000 \text { brightest } \\
\text { (CCD) stars. The ASC database for initial } \\
\text { attitude acquisition contains the } 2000 \\
\text { brightest stars. }\end{array}$} \\
\hline
\end{tabular}

using the moon as the blinding object. However, due to the very tight schedule, on Teamsat we could only fly one CHU and one DPU.

The non-stellar object detector was also assessed in real-sky tests using earth-orbiting satellites as objects. At a typical pointing accuracy of 3 arcsec absolute object tracking noise was less than 25 arcsec. These results were achieved with a star detection limit of $m_{v}$ 9. The non-stellar object function may be enabled by command, and the number of objects tracked (up to 200) is user selectable.

\section{Hardware design reliability}

Throughout the design of the HW, the emphasis has been put on accuracy, functionality, low power, thermal stressing, 
and mass and size. SMD technology has been utilized, increasing the board density and maximizing the mechanical stability. This has led to a compact design with a very low IC-count. In some cases commercial chips proved by far the best and, in some cases, the only choice, e.g. the CCD-chip.

Due to this design procedure, all chips used in the design have been thoroughly screened for radiation tolerance with respect to total-dose, dose-rate for Single Event Upset (SEU) and Single Event Latch-up (SEL). If a candidate chip in this process, failed to sustain $30 \mathrm{KRad}$ on-chip dose, proved latch-up prone at LET's below $20 \mathrm{MeV}^{*} \mathrm{~cm}^{2} / \mathrm{mg}$ or exhibited a high SEU rate, the chip was excluded from use. After the acceptance screening, a number of chips from the same batch are acquired and the tests performed again on samples from each batch to confirm the validity of the screen-tests for that batch.

The above mentioned levels ensures correct operation during exposure to solar wind and trapped protons (Van Allen belts), to cope with SEL's generated by the rare cosmic particles, each circuit block is protected by individual latch-up protection circuitry.

Thermally, the design has been verified to conform to the design, so as no component has a temperature that deviates more than $14^{\circ} \mathrm{C}$ from the box surface in vacuum.

The compact design and the miniaturization provide for a rugged design with high resonant frequencies, resulting in high shock and vibration level tolerances. The $40 \mathrm{~g}$ (sine) vibration tolerance prototype level has been verified, and flight instrument testing at various launcher profiles sustains this.

After assembly, the circuitry is tested for function and parametric variations, upon which a burn-in procedure is performed. Accelerated life tests are performed on sample boards from the batch.

Throughout the design emphasis has been put on making system performance independent of the degradation of various parameters with age, or radiation doses. E.g. the autonomous offset and gain control in the analog signal path from the camera is able to cope with large variations in the CCD-chip sensitivity, the Correlated Double Sampler sensitivity or the line amplification. Other examples are the SW-maintained hot-spot database that handles radiation induced (temporary or static) defects in the $\mathrm{CCD}$, full utilization of the inherent $\mathrm{HW}$ protection mechanisms in the processor used to trap SEU's, and full in-flight upload capability of the SW except from a small core loader.

It is important to stress that this design is not latch-up free. Latch-up may occur but the hardware and software protection mechanisms detect them and reboot the instrument recovering the nominal operations in few seconds.

\section{Simulation mode}

Another interesting feature of the AVS is the built-in simulation mode. This mode allows simulating the performances of the instrument to the highest degree of fidelity in closed loop with the spacecraft or testbeds.

\section{THE AVS MISSION}

The Ariane 502 launch provided an excellent opportunity to test the AVS performances in space and to gather data about the radiation effects. Indeed, all the capabilities of the vision system could be tested and validated. The identification and tracking functions could have used the speltra ${ }^{1}$, YES and above all the geostationary satellites as possible targets. In particular if the YES could have been tracked after ejection we could have obtained unique visual and dynamic information of the relative motion. The non-stellar objects might be tracked from image to image, and as the line of sight to each is known in inertial reference frame coordinates, their orbital elements may be determined. Also, the instrument could be used as a science camera detecting asteroids in a deep-space mission.

Attitude measurements could have been used throughout the whole mission to monitor the Teamsat attitude, attitude rate and stability. This was quite important and, scientifically interesting too, since Teamsat was an unstable spinner. These measurements could have been useful, in case of anomalous behaviour, to decide when YES should have been ejected ${ }^{2}$.

Due to the highly eccentric orbit, the experiment periodically crossed the Van Allen proton belts. The imaging and compression capabilities were used to assess and to analyse the transient and quasi-stationary effects of the high radiation environment on the image generation. The latter to allow for analysis of the impact in the image quality or degradation of the CCD. This was done on ground by comparing images taken before, during and after each crossing of the radiation belts.

The Teamsat instrument was mechanically slightly different from the one described in the previous because, due to the short time available to build the instrument, we had to reuse spare flight components and also because we were interested to gather data about the radiation, hence we reduced the shielding. The instrument that flew on Teamsat was composed of two units:

\footnotetext{
${ }^{1}$ The speltra, shown in fig. 2 , is a structure, used in the Ariane 5 dual launches, to carry the upper payload and to protect the lower passanger.

2 This issue was extensively analysed until the decision not to deploy YES was taken. Without the tether the orbit into which YES was injected was of much less concern.
} 


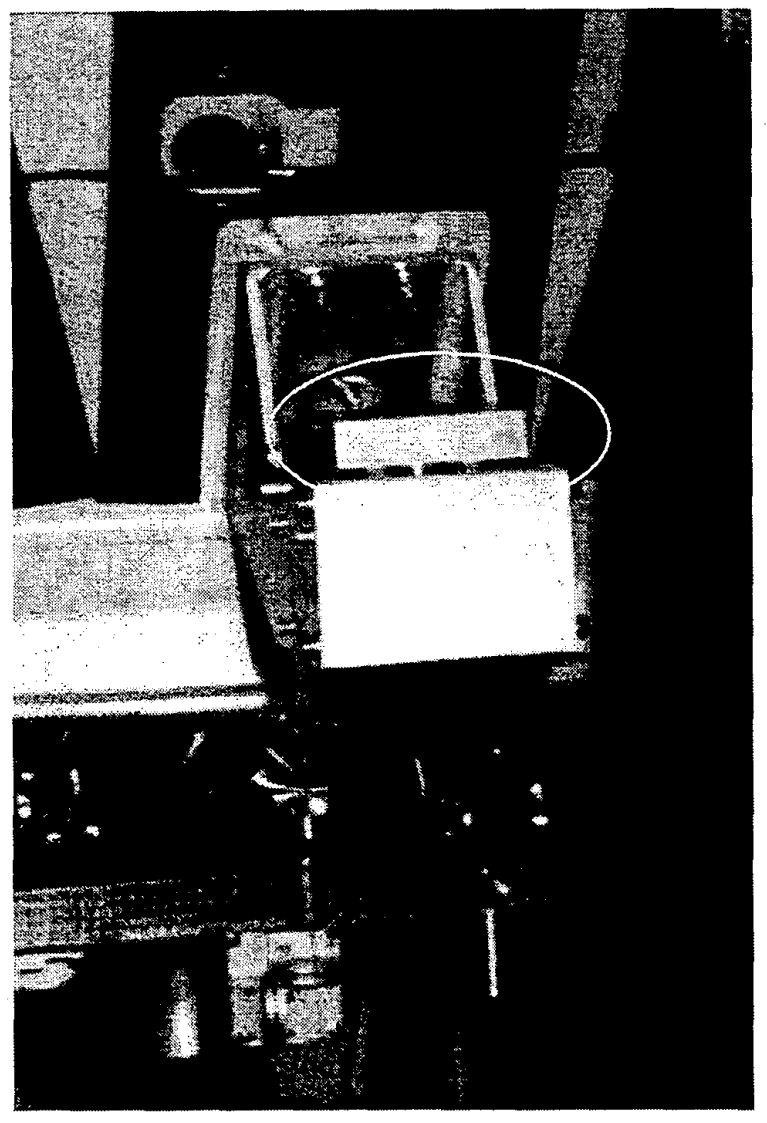

Figure 5: The AVS CHU mounted on the bracket on the MAQSAT-H cone. The baffle, mounted inside the bracket, is not visible.

1. A $180 \mathrm{~g} 0.4 \mathrm{~W}$ CCD camera (fig. 5) with a very short, 5 $\mathrm{cm}$, single stage baffle;

2. A 486-based 700g 5W Data Processing Unit (fig. 6).

The camera was mounted parallel to the spinning axis pointing backwards.

\section{MISSION RESULTS}

Due to a launcher roll anomaly, TeamSat was injected with a velocity deficit of $210 \mathrm{~m} / \mathrm{s}$ into a GTO orbit with the parameters given in table 2 .

Table 2: TeamSat achieved and nominal orbital parameters

\begin{tabular}{|l|r|r|}
\hline Parameter & \multicolumn{1}{|c|}{ Achieved } & Nominal \\
\hline Semi-major axis (Km) & 20140 & 24661 \\
\hline Eccentricity & 0.657 & 0.718 \\
\hline Inclination $\left(^{\circ}\right.$ ) & 7.76 & 7.75 \\
\hline $\begin{array}{l}\text { RAAN }(\text { Right Ascension } \\
\text { of the Ascending Node }\left({ }^{\circ}\right)\end{array}$ & 58 & 58 \\
\hline Argument of perigee $\left({ }^{\circ}\right)$ & 178 & 178 \\
\hline
\end{tabular}

Furthermore, as the propellant was used to compensate the roll anomaly and to increase the apogee altitude, the upper

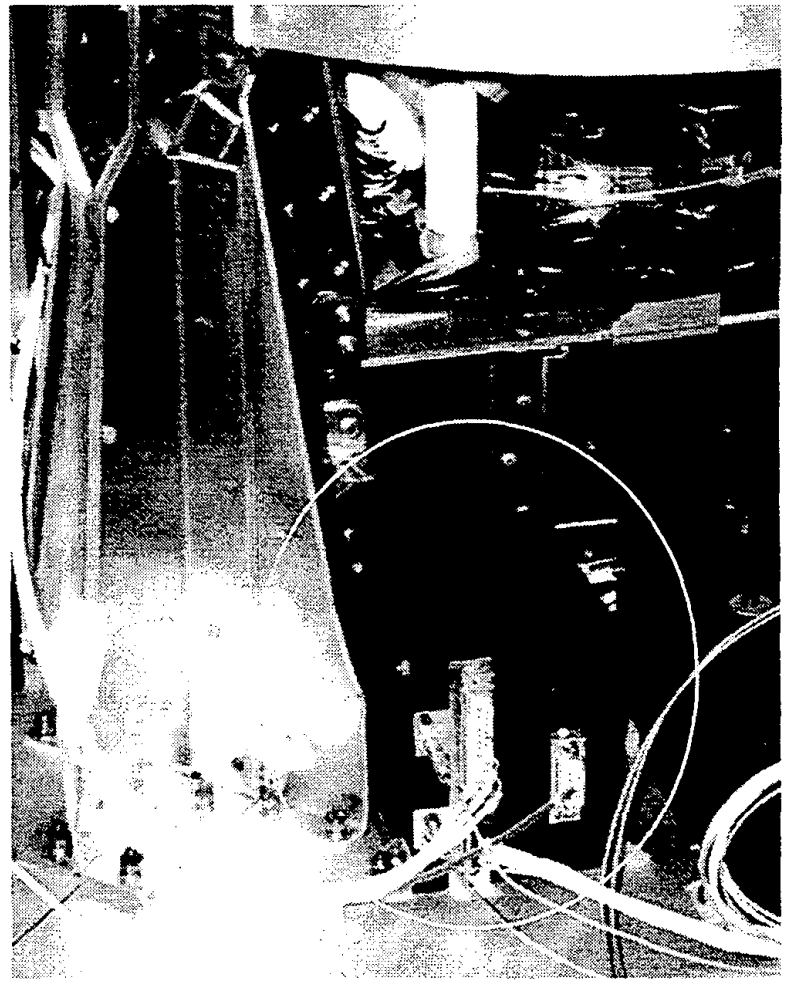

Figure 6: The AVS DPU mounted inside the TeamSat box and electrically connected.

stage did not spin-up and oriented properly TeamSat before release. Hence, TeamSat was released with unknown attitude parameters (table 3 shows the nominal attitude).

Table 3: TeamSat nominal attitude

\begin{tabular}{|l|c|}
\hline Parameter & Nominal Value \\
\hline $\begin{array}{l}\text { TeamSat angular momentum pointing } \\
\text { direction: } \alpha\left(^{\circ}\right)\end{array}$ & 356.22 \\
\hline $\begin{array}{l}\text { TeamSat angular momentum pointing } \\
\text { direction: } \delta\left(^{\circ}\right)\end{array}$ & -6.84 \\
\hline spin rate (rpm) & 0.5 \\
\hline
\end{tabular}

The non-nominal dynamic conditions (both orbit and attitude) posed a few problems soon after the launch because we were not aware of the non-nominal attitude and hence we were not able to initially match the AVS output with the expected attitude. Nevertheless, the instrument worked perfectly proving its performance and robustness beyond expectations.

All the mission objectives were successfully reached. The non-stellar object tracking capabilities and the radiationinduced effects are treated in detail in [1] and [2] respectively.

The autonomy worked perfectly though some parameters had to be changed in-flight because of the rather anomalous attitude dynamics. 


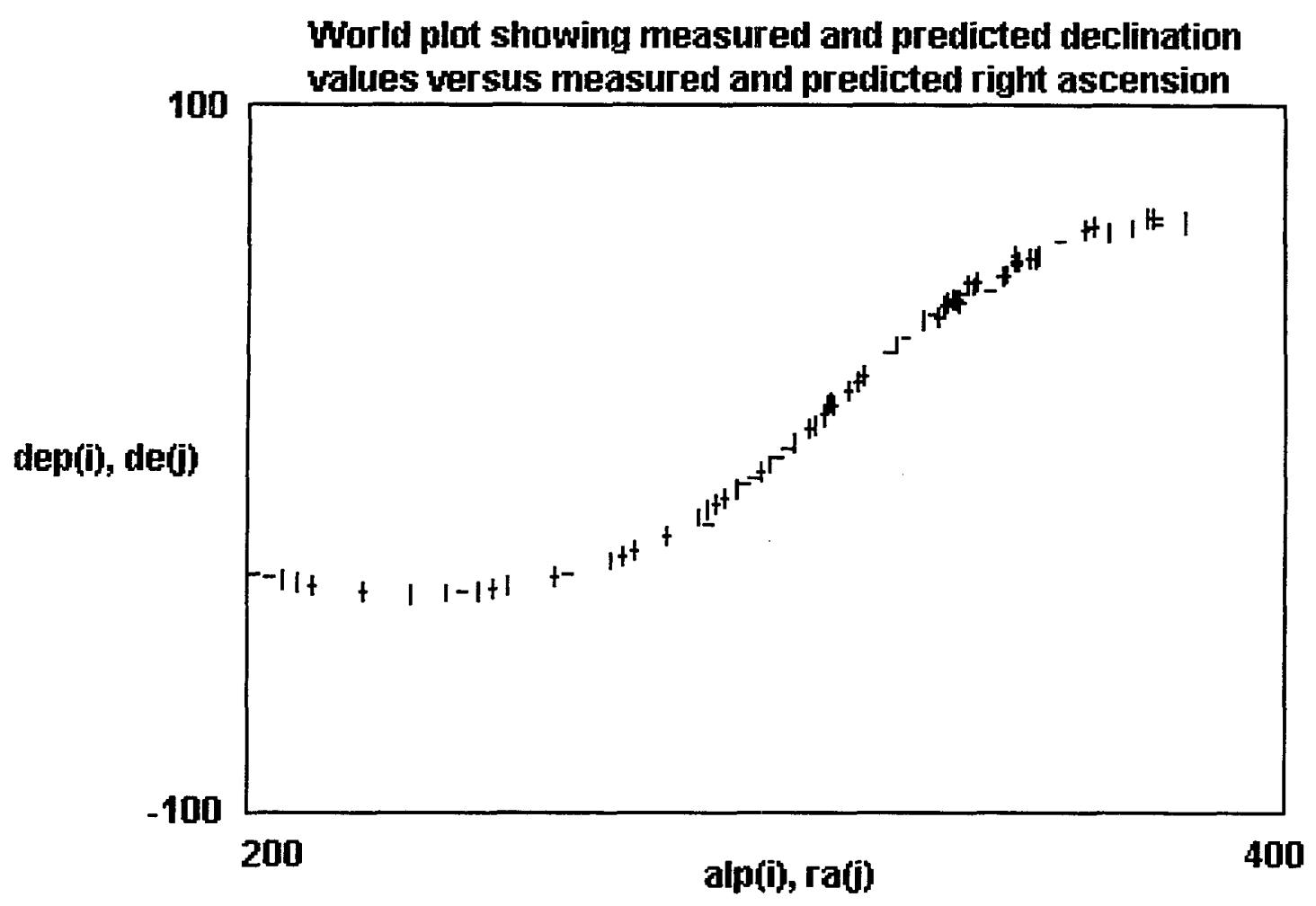

Figure 7: AVS attitude measurements and model fit (world plot)

A major achievement of the AVS was the determination of the TeamSat attitude dynamics. The accurate attitude measurements provided by the instrument indeed allowed reconstructing the TeamSat attitude dynamics. Fig. 7 shows the instrument measurements and the sinusoidal fit. The parameters derived by the fitting are shown in fig. 7, 8 and 9.

From the figure and the data (the Sun ephemeris were $\alpha_{\text {Sun }}=$ $215^{\circ}$ and $\delta_{\text {Sun }}=-14^{\circ}$ ) it can be seen that AVS was blinded for most of the time by the Sun light and that for longer period of time the Sun light was shining directly on the CCD. In this respect the robustness of the AVS design was severely and successfully tested. Indeed, not only the CHU was not damaged, permanently or temporarily by the direct Sun light, but the AVS restored nominal operations as soon as the Sun was not in the instrument field of view.

\section{CONCLUSIONS}

The sensor performed very well throughout the whole mission from the mechanical, electrical, optical and $\mathrm{S} / \mathrm{W}$ point of views. Although the CCD was exposed to the harsh space environment, i.e. sun light, large temperature variations and more than twenty crossing of the proton belt and constantly embedded in the electron belt, almost nominal operation was achieved.

The performances are even more remarkable if we consider that this unit was built in only a few weeks.

The attitude was determined very accurately, despite the fact that attitude dynamics were quite different from the nominal ones for which the on-board parameters were set, and the parameters defining the TeamSat attitude dynamics were defined.

Several images were taken showing both non-stellar objects and the effects induced by the high radiation.

One last remark about TeamSat's final moments. The power estimate was too pessimistic and indeed, after more than twenty orbits of intensive operations, the batteries were still alive. Therefore we decided to switch on AVS again just in time to gather few nice more images with some bright nonstellar objects in view. Then, the voltage started to drop, the transponder coughed and with only five people witnessing the event, after three days in orbit, TeamSat died! 


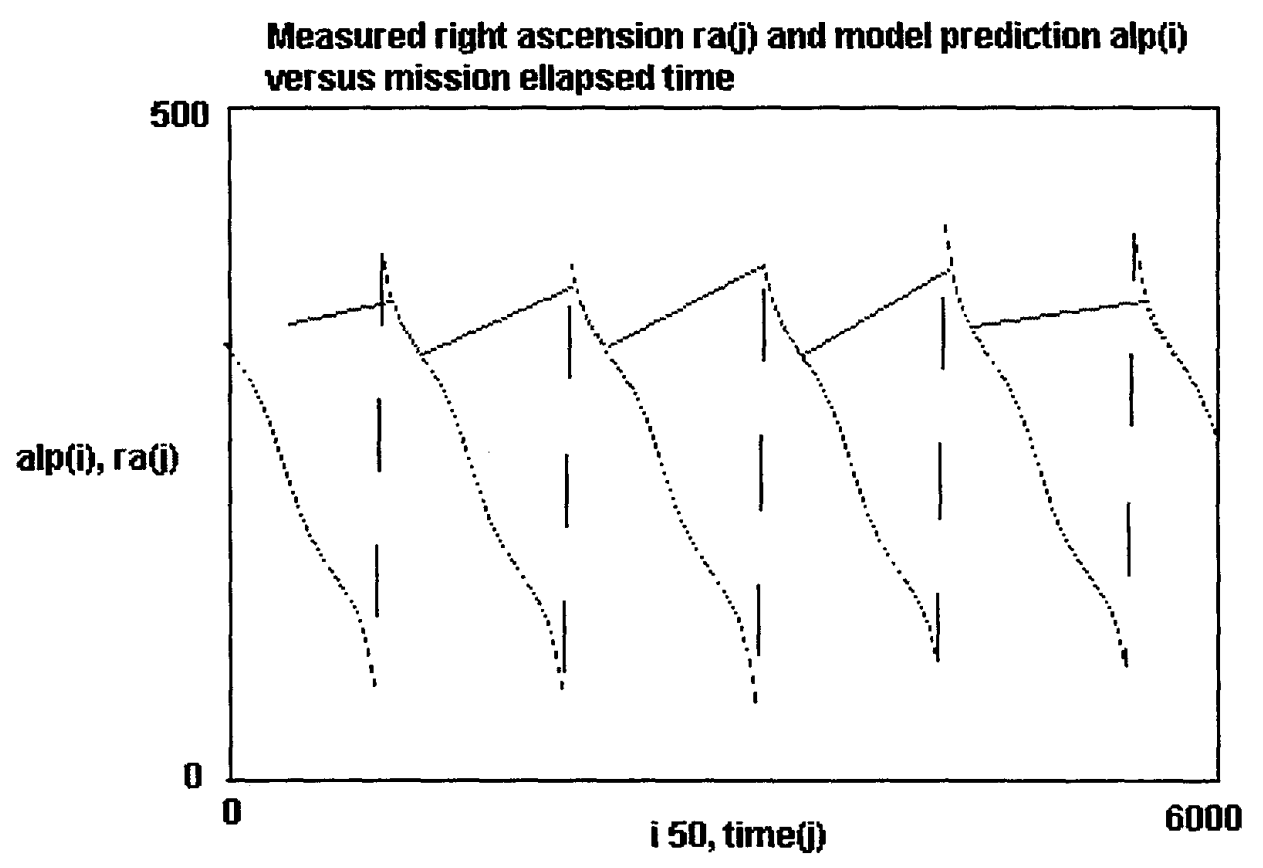

Figure 8: AVS right ascension measurements and model fit

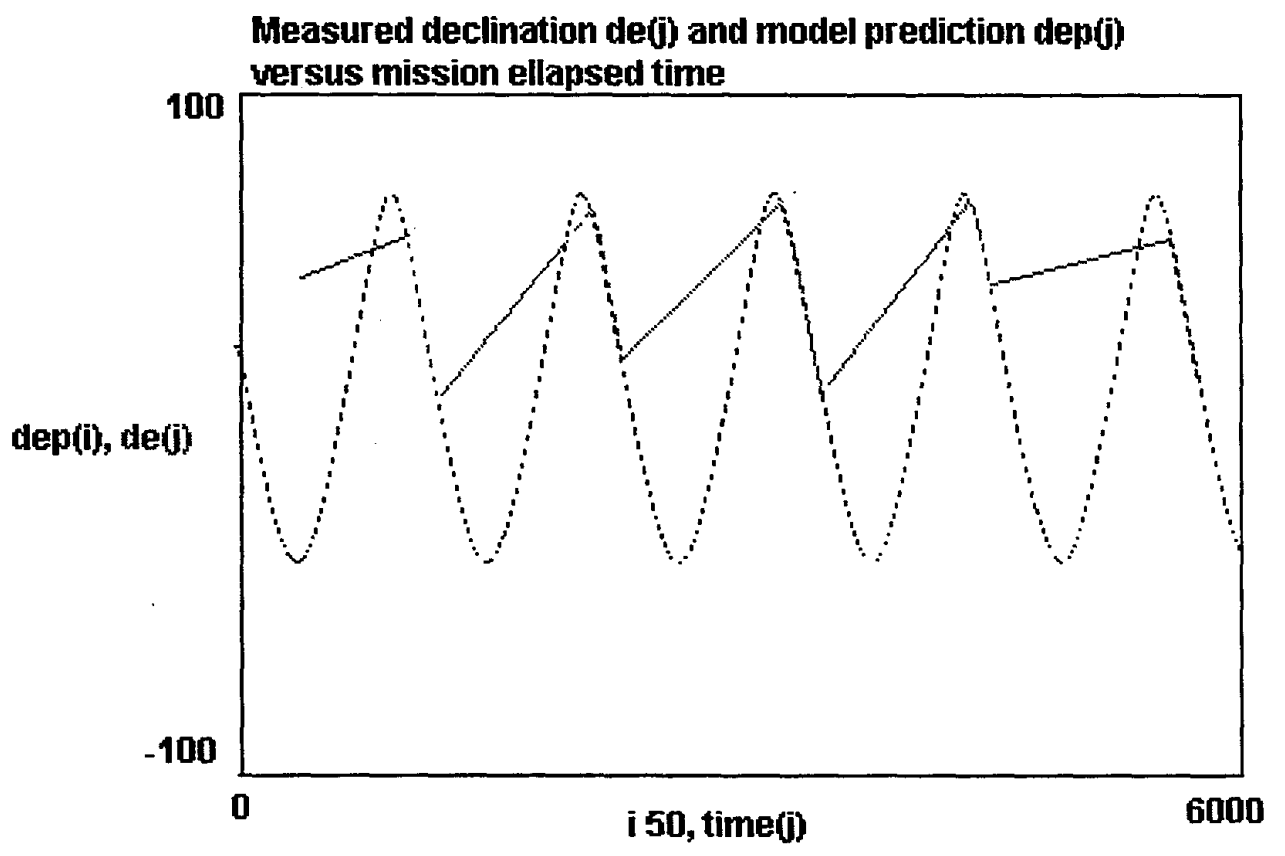

Figure 9: AVS declination measurements and model fit 


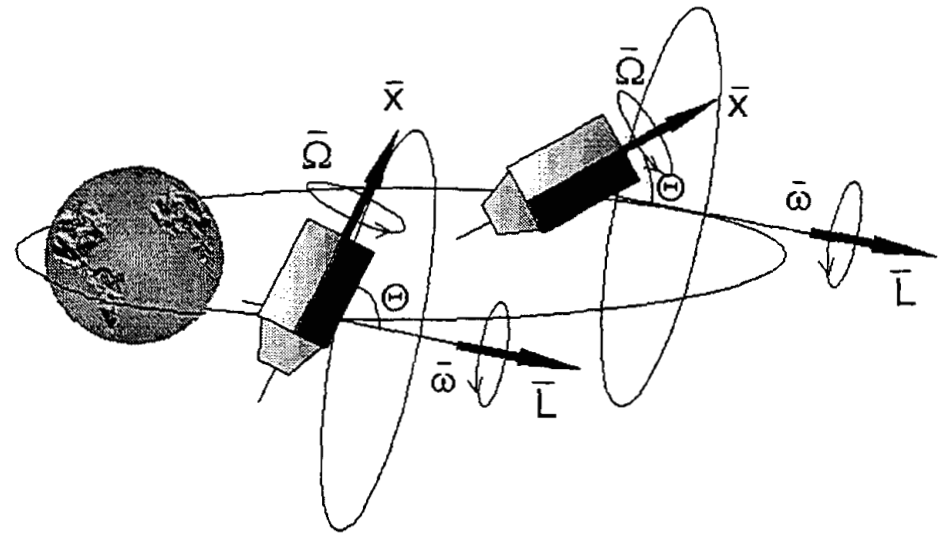

$$
\begin{array}{rlrl}
\text { R.A.L } & =52 \mathrm{deg} & \omega & =0.315 \mathrm{deg} / \mathrm{sec} \\
\text { DEC. }_{\mathrm{L}}=-36 \mathrm{deg} & \Omega & =7.8856 * 10^{-2} \mathrm{deg} / \mathrm{sec} \\
\Theta & =74 \mathrm{deg} & \phi & =20 \mathrm{deg} \text { at MET: } 40787 \text { (not defined in the figure) }
\end{array}
$$

Figure 10: The TeamSat attitude dynamics parameters as derived by the AVS measurements fit and their graphical representation.

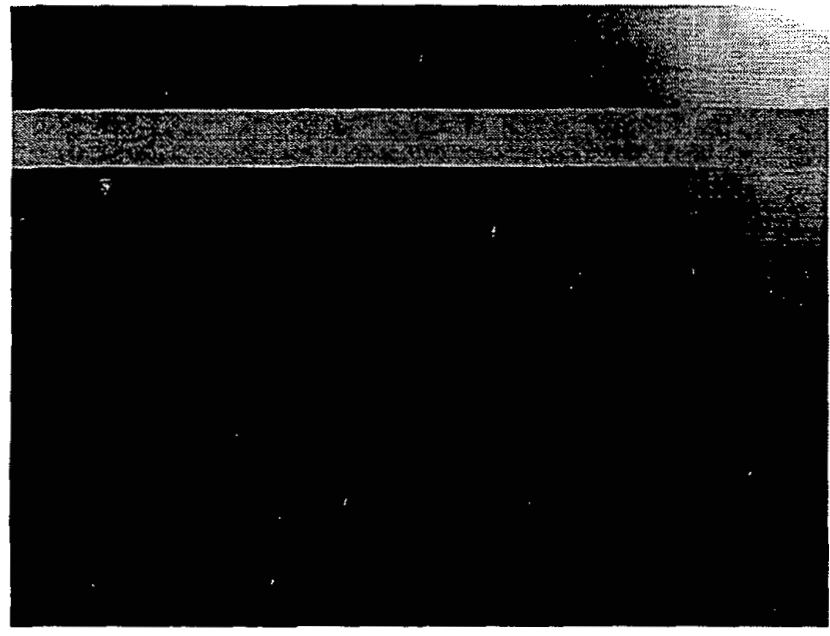

Figure 11: The first image taken by the AVS. Note the Earth in the upper right corner, the dark background, the lack of hot spots and one non-stellar object on the upper left area (the bright spot below the TM drop-out).

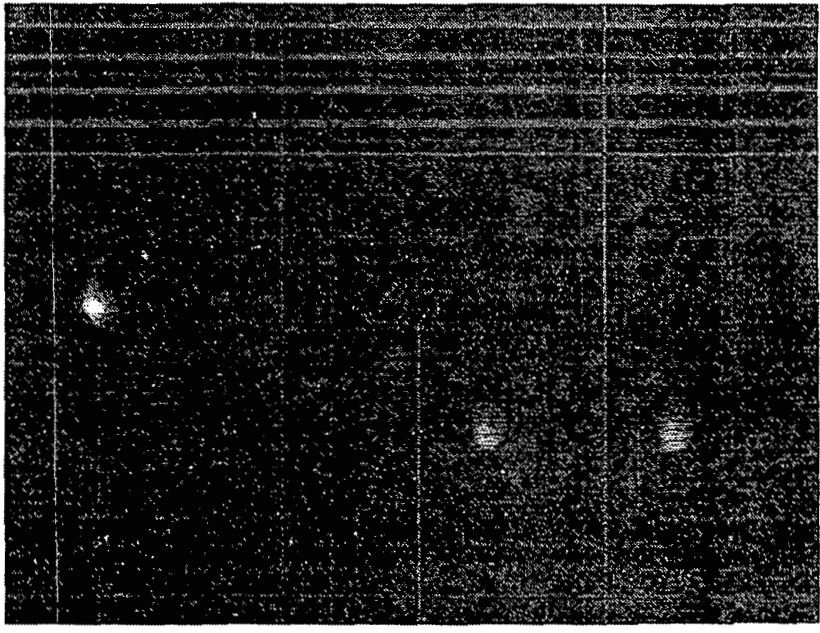

Figure 12: The last image taken by the AVS. Note the increased background level, the numerous bright spots (hot spots and fireflies) and two bright non-stellar objects. The objects in the bottom right corner, clearly the same, are due to a transmission error. 


\section{ACKNOWLEDGEMENTS}

We would like to thank the European Space Agency (ESA), the Centre Nationale d'Etudes Spatialle (CNES) and the Arianespace for having granted us this opportunity.

Furthermore we would like to thank Mr. Ockles, Mr. Bandecchi and the whole Team for their enthusiasm and dedication, without which this project would not have happened.

\section{REFERENCES}

[1] T. Riis, J.L. Jørgensen, M. Betto, "Operations of a nonstellar object tracker in space," IEEE Aerospace Conference, 1999.

[2] J.L. Jørgensen, T. Riis, M. Betto, "Star tracker and vision system performance in a high radiation environment," IEEE Aerospace Conference, 1999.

[3] J.L. Jørgensen, C.C. Liebe, "The Advanced Stellar Compass, Development and Operations," Acta Astronautica, Vol.39, Nov-Dec. 1996.

[4] M.M. Birnbaum, "Spacecraft Attitude Control using Star Field Trackers," Acta Astronautica, Vol.39, NovDec. 1996.

[5] M. Bandecchi, W.J. Ockles, "The TeamSat Experience" ESA bulletin 95, Aug. 1998.

[6] A. Bradford, F. Müller-Stute, B. Sarti, "Engineering TeamSat - From Concept to Delivery," ESA bulletin 95, Aug. 1998.

[7] S. Habinc, D. Hardy, P. Sinander, C Smith, "TeamSat's Data Handling System," ESA bulletin 95, Aug. 1998.

[8] M. Jones, B. Melton, M. Bandecchi, "TeamSat's lowcost egse and mission control system," ESA bulletin 95, Aug. 1998.

Maurizio Betto is currently a PhD student at the Technical University of Denmark (DTU) in the Space Instrumentation Group (SIG) working on the navigation function of the AVS. He has a MSc in Astronomy and worked seven years at the European Space Agency in the field of the guidance navigation

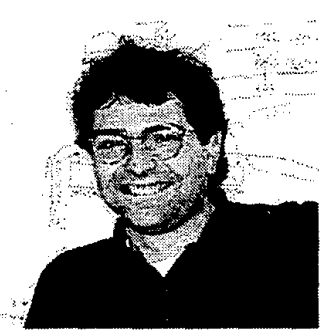

John L. Jørgensen is a professor at the department of Automation at the Technical University of Denmark (DTU), where he is head of the Space Instrumentation Group (SIG) that developed the AVS. He has a MSc in Engineering and a HD in business and administration. His main research activities are

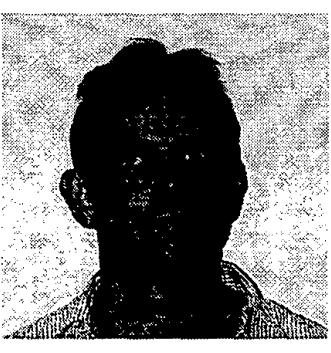
robust vision systems, star trackers and vision in space.

Troels Riis is currently a Ph.D. student at the Technical University of Denmark (DTU) in the Space Instrumentation Group (SIG). He has a MSc in Engineering. $\mathrm{He}$ is mainly doing research in the areas of non-stellar object detection and tracking, and pattern recognition for the AVS.

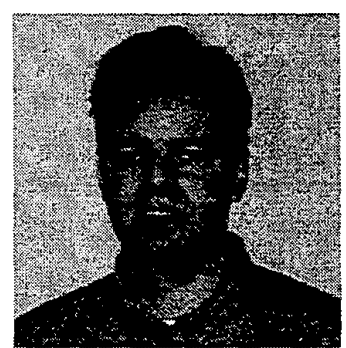
and control. 